\title{
ON THE AUTOMORPHISM GROUP OF A CONNECTED LOCALLY COMPACT TOPOLOGICAL GROUP
}

\author{
by TA-SUN WU
}

(Received 30th July 1990)

\begin{abstract}
Let $G$ be a locally compact connected topological group. Let $A u t_{0} G$ be the identity component of the group of all bi-continuous automorphisms of $G$ topologized by Birkhoff topology. We give a necessary and sufficient condition for Aut $_{0} G$ to be locally compact.
\end{abstract}

1980 Mathematics subject classification (1988 Revision): Primary 22A05.

Let $G$ be a connected locally compact (Hausdorff) topological group. Let Aut $G$ denote the group of all bi-continuous automorphisms of $G$ topologized by Birkhoff topology $\left(\left[1,2,3\right.\right.$, III 3], [4]). Let $\mathrm{Aut}_{0} G$ be the identity component of Aut $G$. In general, Aut $_{0} G$ is not locally compact. The purpose of present note is to give a necessary and sufficient condition so that $\mathrm{Aut}_{0} G$ is a locally company group. Precisely, we show the following condition holds:

Theorem. Let $G$ be a connected locally compact group. Let Aut $G$ be the group of all bi-continuous automorphisms of $G$ topologized by Birkhoff topology. Let Aut $G$ be the identity component of Aut $G$. Then $\mathrm{Aut}_{0} G$ is locally compact if and only if one of the following two conditions holds.

(1) The dimension of the centre $Z(G)$ of $G$ is finite.

(2) The closure of the commutator subgroup of $G$ is uniform in $G$, i.e. $G /[G, G]^{-}$is compact and Aut $G$ is a Baire space, i.e. it satisfies the Baire Category Theorem.

When $\mathrm{Aut}_{0} G$ is locally compact, $\mathrm{Aut}_{0} G$ is the direct product of an analytic group and a compact connected group with trivial centre.

We note when $G$ is a second countable locally compact group, then Aut $G$ is a Baire space. Aut ${ }_{0} G$ is a closed subgroup of Aut $G$. It is also a Baire space ([11]).

In [1], it was shown Aut $_{0} G$ is locally compact if $G$ is a compactly generated locally compact group and $\mathrm{Aut}_{0}\left(G_{0}\right)$ is locally compact. So our results here cover the case when $G$ is a compactly generated locally compact group.

The proof of the above theorem will be preceeded by a sequence of lemmas. Some of them have their own interests.

From now on, $G$ will be a connected locally compact Hausdorff topological group. It 
is well known that $G$ has maximal compact subgroups ([6]). Maximal compact subgroups of $G$ are conjugated by inner automorphisms and they are connected. Every compact normal subgroup of $G$ is contained in every maximal compact subgroup. There exists a (unique) maximal compact normal subgroup $K$ of $G . G$ is a pro-Lie group and $G / K$ is an analytic group.

Lemma 1. Let $F$ be the identity component of the centralizer of $K$ in $G$. Then $G=F K$. Both $F$ and $K$ are characteristic subgroups of $G$. Let $Q=F \cap K$. Then $Q$ is the maximal compact subgroup of the centre of $F$. Furthermore, $Q$ is the maximal compact normal subgroup of $F$.

Proof. Let $F^{\prime}$ be the centralizer of $K$ in $G$, i.e. $F^{\prime}=\{x \in G: x k=k x$ for all $k \in K\}$. Then $G=F^{\prime} K$ (cf. [6, Theorem 2]). We have the following natural isomorphism $F^{\prime} / F K \cap F^{\prime} \rightarrow F^{\prime} K / F K$. Since $F K \cap F^{\prime}$ contains the identity component $F$ of $F^{\prime}$, hence $F^{\prime} / F K \cap F^{\prime}$ is totally disconnected unless $F^{\prime}=F K \cap F^{\prime}$. Since $F^{\prime} K / F K=G / F K$ is connected, therefore $F^{\prime}=F K \cap F^{\prime}$ and $G=F^{\prime} K=F K$. It is clear that $K$ is a characteristic subgroup of $G$. $F$ is the centralizer of $K$, hence $F$ is also characteristic in $G$. Let $Z(F)$ be the centre of $G . Z(F)$ is compactly generated, so $Z(F)$ has a (unique) maximal compact subgroup $X$. Then $X \subset F \cap K$ since $X$ is compact and normal in $G$. On the other hand $F \cap K$ is central in $G$. Therefore $F \cap K=X$. Finally, let $Y$ be the maximal compact normal subgroup of $F$. Since $Y$ is a characteristic subgroup of $F$, it is normal in $G$ (in fact, characteristic in G). Hence $Y \subset K$. We have $X \subset Y \subset F \cap K=X$. We conclude $Q=F \cap K$ is also the maximal compact normal subgroup of $F$. The proof of the lemma is now complete.

We preserve all the notation in the above lemma throughout the rest of this note.

Lemma 2. Let $\sigma$ be any automorphism from Aut $_{0} G$. The restriction of $\sigma$ to $Q$ is an identity map.

Proof. Since $K$ is a characteristic subgroup, we have the restriction map $\pi:$ Aut $G \rightarrow$ Aut $K$. Then $\pi\left(\right.$ Aut $\left._{0} G\right)$ is a connected subgroup of Aut $K$. Since Aut $K / \operatorname{Inn} K$ is totally disconnected $([6$, Theorem 1]), therefore $\pi(\sigma)=\sigma / K$ is an inner automorphism. Because $Q$ is central in $K, \pi(\sigma)=\sigma / Q$ is the identity map.

Proposition 3. $\mathrm{Aut}_{0} G \cong \mathrm{Aut}_{0} F \times \mathrm{Aut}_{0} \mathrm{~K}$.

Proof. Let $\tau$ be any automorphism of $F$ from the identity component of Aut $F$. Since $Q$ is a characteristic subgroup of $F$, so $\tau(Q)=Q$. By the same argument as in Lemma 2, the restriction of $\tau$ to $Q$ is the identity map. Hence, we can extend $\tau$ to an automorphism $\tau^{\prime}$ of $G$ simply by defining $\tau^{\prime}(x k)=\tau(x) k$ for $x \in F$ and $k \in K$. Similarly, for every $\sigma \in \mathrm{Aut}_{0} K, \sigma$ can be extended to an automorphism $\sigma^{\prime}$ of $G$ by $\sigma^{\prime}(x k)=x \sigma(k)$. Hence $A_{u t} G$ is isomorphic to the direct product of $A_{u t} F$ and $A_{0} t_{0} K$. 
Corollary 4. Aut ${ }_{0} G$ is locally compact if and only if $\mathrm{Aut}_{0} F$ is locally compact.

Proof. Since $\mathrm{Aut}_{0} K$ is compact, $\mathrm{Aut}_{0} G$ is locally compact if and only if $\mathrm{Aut}_{0} F$ is locally compact.

We quote the following results for reference.

Lemma 5. [7]. If $\operatorname{dim} Q$ is finite, then $F$ is a finite-dimensional connected locally compact group and Aut $F$ is a Lie group.

Lemma 6. Let $[F, F]$ be the subgroup of $F$ generated by the commutators of $F$. If $\operatorname{dim} Q$ is not finite and $\mathrm{Aut}_{0} F$ is locally compact, then $F /[F, F]^{-}$is compact.

Proof. $F /[F, F]^{-}$is isomorphic to the product of a vector group $V$ and a compact connected abelian group. If $F /[F, F]^{-}$is not compact, $V$ is not trivial. Then $F$ is a semi-direct product of a normal subgroup $F_{1}$ and a one parameter subgroup $R, F=$ $F_{1} \cdot R$. Let $\phi$ be any continuous homomorphism from $R$ into $Q$. Define the automorphism $\sigma_{\phi}$ of $F$ by $\sigma_{\phi}(x r)=x r \phi(r)$ where $x \in F_{1}$ and $r \in R$. When $Q$ is an infinite dimensional compact abelian group, given any integer $n$, there exists a compact subgroup $Q_{n}$ such that $Q / Q_{n}$ is isomorphic with the $n$-dimensional torus group $T^{n}$. Thus for every $n$, Aut ${ }_{0} F / Q_{n}$ contains a closed subgroup $V_{n}^{\prime}$ which is isomorphic with the $n$-dim vector group $R^{n}, V_{n}^{\prime}=\left\{\sigma_{\phi^{\prime}}: \phi^{\prime} \in \operatorname{Hom}\left(R, T^{n}\right)\right\}$. Let $\delta_{n}$ be the quotient map from $Q$ onto $Q / Q_{n}$. Define $\delta_{n}^{*}$ from $\operatorname{Hom}(R, Q)$ to $\operatorname{Hom}\left(R, Q / Q_{\pi}\right)$ by $\delta_{n}^{*}(\phi)=\delta_{\pi} \circ \phi$. Since every homomorphism from $R$ into $T^{n}$ can be lifted to a homomorphism from $R$ into $Q$, (cf. [10]), therefore $\delta_{\pi}^{*}$ is an epimorphism. Because $Q_{\pi}$ is a subgroup of $Q$ where $Q$ is pointwise fixed by Aut $_{0} F$, there is a natural homomorphism $\pi_{n}$ from Aut $_{0} F$ into Aut $_{0} F / Q_{n}$. Let $V_{n}=\left\{\sigma_{\phi}: \phi \in \operatorname{Hom}(R, Q)\right\} \subset \operatorname{Aut}_{0} F$. Since $\delta_{n}^{*}$ is an epimorphism, $\pi_{n}\left(V_{n}\right)=$ $V_{n}^{\prime}$ for every $n$. This shows that for each integer $n, \mathrm{Aut}_{0} F$ has a homomorphic image which contains a $n$-dim vector group. When $A t_{0} F$ is locally compact, this is impossible. Therefore we conclude that $F /[F, F]^{-}$must be a compact group. The proof is complete.

Corollary 7. Let $M$ be a maximal compact subgroup of $F$. If $F /[F, F]^{-}$is compact then $F=[F, F] M$.

Proof. Let $\pi$ be the canonical homomorphism from $F$ onto $H=F / Q$. Then $\pi(M)$ is a maximal compact subgroup of $H$. Observe that $H$ does not have non-trivial compact normal subgroup. Hence the nilradical $N$ of $H$ is simply connected. Let $R$ be the radical of $H$. Let $N^{\prime}$ be the radical of $[H, H]$. Then $N^{\prime}$ is a simply connected normal closed subgroup of $N$. By Proposition $6, R / N^{\prime}$ is compact. Thus $R \subset N^{\prime} \pi(M)$. Then $H=[H, H] \pi(M)$. And we have $F=[F, F] M$ as desired.

8. Since $F$ is a connected locally compact group, locally $F$ has a direct product decomposition. This means there exist a local Lie group $L$ and a compact normal 
subgroup $D$ such that $[L, D]=(1)$ and $L D$ is a neighbourhood of 1 in $F$. Since $F$ is connected, $F=\bigcup_{n=1}^{\infty}(L D)^{n}=\left(\bigcup_{n=1}^{\infty} L^{n}\right) D=\left(\bigcup_{n=1}^{\infty} L\right) Q$. Let $\mathscr{L}=\bigcup_{n=1}^{\infty} L$. Then $[F, F]=$ $[\mathscr{L} Q, \mathscr{L} Q]=[\mathscr{L}, \mathscr{L}]$. So $[\mathscr{L}, \mathscr{L}]$ is a characteristic analytic subgroup of $F$. Assume now that $F /[F, F]^{-}$is compact. Because $M$ is a maximal compact subgroup. $[F, F]^{-} \mathrm{M}=$ $[\mathrm{F}, \mathrm{F}] M=F$. Therefore $F=[\mathscr{L}, \mathscr{L}] M$. Since $F=\mathscr{L} Q, \mathscr{L}$ is a normal subgroup.

Lemma 9. Let $L \times D$ be a neighbourhood of 1 in $F$ with $L$ a local Lie group and $D a$ compact subgroup. Let $\mathscr{L}=\bigcup_{n=1}^{\infty} L^{n}$. Assume $F /[F, F]^{-}$is compact. Then every element $s \in \mathscr{L}$ can be expressed as $s_{1} s_{2}$ with $s_{1} \in[\mathscr{L}, \mathscr{L}]$ and $s_{2}$ a compact element.

Proof. By Corollary $7, F=[F, F] M=[\mathscr{L}, \mathscr{L}] M$. Let $s \in \mathscr{L}$. Then $s=s_{1} s_{2}$ for some $s_{1} \in[\mathscr{L}, \mathscr{L}]$ and $s_{2} \in M$. Then $s_{2}=s_{1}^{-1} s \in \mathscr{L} \cap M$. Hence $s_{2}$ is a compact element in $\mathscr{L}$.

We need some information about the space of all maximal compact subgroups of $F$. The following fact is known ([8, Section 5.3]): Let $H$ be a Lie group and $P$ be a compact subgroup of $H$. Then there exists an open set $O$ in $H, P \subset O$ with the property that if $P^{\prime}$ is a compact subgroup of $H$ and $P^{\prime} \subset O$, then there is an element $h$ in $H$ such that $h^{-1} P^{\prime} h \subset P$. Moreover given any neighbourhood $W$ of the identity of $H, O$ can be so chosen that for every $P^{\prime} \subset O$ the desired $h$ can be selected in $W$.

Now let us assume $H$ is an analytic group. So $H$ has maximal compact subgroups. Let $\mathscr{Y}$ be the space of all the maximal compact subgroup of $H$. Let $P \in \mathscr{Y}$. Let $\mathscr{N}_{H}(P)$ be the normalizer of $P$. Since any two maximal compact subgroups of $H$ are conjugate by an inner automorphism, in view of above observation, it is natural to identify $\mathscr{Y}$ with the left coset space $H / \mathscr{N}_{H}(P)$. Now we shall apply these results to the group $F$. Let $\underline{X}$ be the space of all maximal compact subgroups of $F$. Let $\pi$ be the canonical map from $F$ onto $H=F / Q$. Since every maximal compact subgroup of $F$ contains the maximal compact normal subgroup $Q$, so $\pi(X)=\mathscr{Y}$. Let $M \in \underline{X}$. Then $X$ can be identified with $F / \mathscr{N}_{F}(M)$. Observe that $F / \mathscr{N}_{F}(M)$ is homeomorphic with $H / \mathscr{N}_{H}(\pi M)$ ). It is a homogeneous analytic manifold.

Lemma 10. Assume that $[F, F]^{-}$is uniform in $F$. Let $M$ be a maximal compact subgroup of $F$. Let $\tau \in$ Aut $_{0} F$. Then there exists an element $f$ in $F$ such that the restriction of $\tau$ to $M$ coincides with the restriction of the inner automorphism $I_{f}$ to $M$ defined by $f$. (Cf. Remark 21).

Proof. First we show there is a global cross-section with respect to the fibre space $F$ over $F / \mathscr{N}_{F}(M)$. Let $\pi$ be the canonical homomorphism from $F$ onto $H=F / Q$. Let $M^{\prime}=\pi(M) . M^{\prime}$ is a maximal compact subgroup of $H$. Notice that $H$ is an analytic group without non-trivial compact normal subgroups. Therefore, the nilradical $N$ of $H$ is simply connected. Because $H /[H, H]^{-}$is compact, $H=N \cdot B$ where $B$ is a maximal reductive subgroup of $H$ and $M^{\prime} \subset B$. Let $T$ be the maximal compact central torus of $B$. Let $B_{1}=B / T$ and $M_{1}=M^{\prime} / T$. Then $M_{1}$ is a maximal compact subgroup of the semi-simple analytic group $B_{1}$. It is known that the normalizer $\mathscr{N}_{B_{1}}\left(M_{1}\right)$ is connected. We sketch a proof. Clearly, we may assume that $B_{1}$ is a simple analytic group. Since the last statement is a general statement, temporarily we change the notation to simplify the 
presentation. Let $S$ denote a non-compact simple analytic group and $P$ a maximal compact subgroup of $S$. If $P$ is a trivial subgroup, then the normalizer of $P$ is $S$ itself and the normalizer of $P$ is connected. Now suppose $P$ is non-trivial. Let $Z$ be the centre of $S$. Let $E$ be a maximal compact subgroup of $S / Z$. Since $S / Z$ is a linear simple group, $E$ is its own normalizer and also it is a maximal closed proper subgroup of $S / Z$ (cf. [5, Exercise A.3. Chapter 6]). Let $\pi$ be the canonical map from $S$ onto $S / Z$. Let $E^{\prime}=\pi^{-1}(E)$. By a theorem of G. D. Mostow, every analytic group is homeomorphic to the direct product of a maximal compact subgroup and a Euclidean space (i.e. exponential manifold, cf. [4, Theorem 3.1, Chapter 15]). So $S / Z$ is homeomorphic to $E \times I$ where $I$ is a Euclidean space. During the course of proving Mostow's theorem, it was shown that $S$ is homeomorphic to the product $\pi^{-1}(E) \times I$ (cf. the proof of Lemma 3.3 Chapter 15 of [4]). Thus $E^{\prime}=\pi^{-1}(E)$ is a connected group. It is a covering group of $E$ since the centre $Z$ is discrete. Thus $E^{\prime}$ is a direct product of a compact group $P^{*}$ and a vector group $V$. Clearly, $P^{*}$ is a maximal compact subgroup of $S$. Furthermore, $P^{*}$ is non-trivial since all maximal compact subgroups of $S$ are conjugate by inner automorphisms and $P$ is a non-trivial compact subgroup of $S$ by assumption. We claim $P^{*} \times V$ is the normalizer of $P^{*}$ in $S$. Let $R$ be the normalizer of $P^{*}$ in $S$. Then $P^{*} \times V \subset R$. This implies $E=\pi\left(P^{*} \times V\right) \subset \pi(R)$. Since $\pi\left(P^{*}\right)$ is non-trivial, so $\pi(R)$ cannot be $S / Z$. Otherwise it would contradict the fact that $S / Z$ is a simple analytic group. Hence $\pi(R)=E ;$ a fortiori, $R=P^{*} \times V$. It is connected. Because any two maximal compact subgroups in $S$ are conjugate, therefore the normalizer of $P$ is also connected. Now let us go back to our original notation. We conclude $\mathscr{N}_{B_{1}}\left(M_{1}\right)$ is connected; a fortiori $\mathscr{N}_{B}\left(M^{\prime}\right)$ is connected. Now, let $x=n b$ be any element in $H=N \cdot B$ which normalizes $M^{\prime}$. Then $(n b)(m)(n b)^{-1}=$ $n\left(b m b^{-1}\right) n^{-1} \in M^{\prime} \subset B$ for every $m \in M^{\prime}$. Since $N$ is a normal subgroup of $H$, from the semi-direct product structure of $N \cdot B$, we have $n\left(b m b^{-1}\right) n^{-1}\left(b m b^{-1}\right)^{-1} \in N \cap B$ and $b m b^{-1} \in M^{\prime}$. Therefore $b \in \mathcal{N}_{B}\left(M^{\prime}\right)$ and $n$ commutes with $M^{\prime}$. Let $N^{\prime}=\left\{n \in N ;\left[n, M^{\prime}\right]=1\right\}$. Then $N^{\prime}$ is an analytic subgroup of $N$. So $\mathcal{N}_{H}\left(M^{\prime}\right)=N^{\prime} \mathcal{N}_{B}\left(M^{\prime}\right)$. It is an analytic subgroup. It is known that $H$ is homeomorphic to the direct product of $M^{\prime}$ with a Euclidean space $E$ (the exponential manifold, cf. Chapter 15 of [4]). Since $\mathcal{N}_{H}\left(M^{\prime}\right)$ is an analytic group, $M^{\prime} \subset \mathscr{N}_{H}\left(M^{\prime}\right) \subset H$. There is a global cross-section $\eta^{\prime}: H / \mathscr{N}_{H}\left(M^{\prime}\right) \rightarrow H$ with respect to the fibre space $H \rightarrow H / \mathscr{N}_{H}\left(M^{\prime}\right)$. Since $F / \mathscr{N}_{F}(M)$ is homeomorphic to $H / \mathscr{N}_{H}\left(M^{\prime}\right)$, accordingly we have the global cross-section $\eta: F / \mathcal{N}_{F}(M)$ to $F$.

Now, for each $\tau \in \mathrm{Aut}_{0} F, \tau(M) \in X=F / \mathcal{N}_{F}(M)$. So $I_{\eta(\tau(M))}^{-1} \circ \tau(M)=M$. Observe $\left\{I_{\eta(\tau(M))}^{-1} \circ\right.$ $\left.\tau / M: \tau \in \mathrm{Aut}_{0} F\right\}$ is a connected subset of $\mathrm{Aut}_{0} M$. Hence the restriction of $I_{\eta(\tau(M))}^{-1} \circ \tau$ to $M$ coincides with an inner automorphism defined by an element of $M$; a fortiori, $\tau / M$ coincides with the restriction of an inner automorphism defined by an element $f$ of $F$. Now the proof of the lemma is complete.

Corollary 11. Assume $F /[F, F]^{-}$is compact. Let $L \times D$ be a local direct product of $F$ with $L$ a local Lie group and $D$ a compact normal subgroup of $F$. Let $\mathscr{L}=\bigcup_{n=1}^{\infty} L^{n}$. Then $\mathscr{L}$ is $A u t_{0} F$ invariant.

Proof. By Lemma 9, every element $\ell \in \mathscr{L}$ is the product $\ell_{1} \ell_{2}$ with $\ell_{1} \in[\mathscr{L}, \mathscr{L}]$ and $\ell_{2} \in M$. Since $[\mathscr{L}, \mathscr{L}]$ is a characteristic subgroup of $F$, it is Aut $_{0} F$ invariant. By Lemma 10 , Aut $_{0} F\left(\ell_{2}\right) \in \mathscr{L}$ since $\mathscr{L}$ is normal in $F$. Hence $\mathscr{L}$ is Aut ${ }_{0} F$ invariant. 
Keep all the notation from Corollary 11 . Let $\mathscr{L}^{*}$ be the analytic group which is obtained from $\mathscr{L}$ by adding $L$ as a neighbourhood of 1 in $\mathscr{L}$. Let $\theta$ be the identification map from $\mathscr{L}^{*}$ onto $\mathscr{L}$. Let $\ell$ be an element in $\mathscr{L}$. We shall adopt the convention: $\rho^{*}=\theta^{-1}(\ell)$. Since $F$ is isomorphic with $\left(\mathscr{L}^{*} \times D\right) / \Delta$ where $\Delta=\left\{\left(d^{*}, d^{-1}\right): d \in D \cap \mathscr{L}\right\}$, every automorphism $\sigma^{*}$ of $\mathscr{L}^{*}$ which leaves $\mathscr{D}^{*}=(D \cap \mathscr{L})^{*}$ pointwise fixed defines an automorphism $\sigma$ on $F$ by the rule: $\sigma(\theta)=\theta\left(\sigma^{*}\left(\ell^{*}\right)\right)$ for all $\ell \in \mathscr{L}$ and $\sigma(d)=d$ for $d \in D$. Thus we have a continuous isomorphism $\theta^{*}$ from Aut $\left(\mathscr{L}^{*}, \mathscr{D}^{*}\right)$ into Aut $F$. Here Aut $\left(\mathscr{L}^{*}, \mathscr{D}^{*}\right)$ denotes the group of all the bicontinuous automorphisms of $\mathscr{L}^{*}$ which leaves $\mathscr{D}^{*}$ pointwise fixed and $\theta^{*}\left(\sigma^{*}\right)=\sigma$. It is clear that $\theta^{*}\left(\operatorname{Aut}_{0}\left(\mathscr{L}^{*}, \mathscr{D}^{*}\right)\right) \subset$ Aut $_{0} F$. Now, given any $\tau \in$ Aut $_{0} F$, since $\tau$ leaves $\mathscr{L}$ invariant, so $\tau$ defines an automorphism $\tau^{*}$ of $\mathscr{L}^{*}$ by the rule: $\tau^{*}\left(\ell^{*}\right)=\tau(\ell)^{*}$. We show that $\tau^{*}$ is bi-continuous. Let $V^{*}$ be any compact neighbourhood in $\mathscr{L}^{*}$. Then there exist countably many elements $\left\{\ell_{i}^{*}\right\}$ in $\mathscr{L}^{*}$ such that $\mathscr{L}^{*}=\bigcup_{i=1}^{\infty} \ell_{i}^{*} V^{*}$. Since $\theta$ is continuous, $\theta\left(\ell_{i}^{*} V^{*}\right)$ is compact. Since $\tau$ is continuous, $\tau \circ \theta\left(\ell_{i}^{*} V^{*}\right)$ is compact. Thus

$$
\mathscr{L}^{*}=\bigcup_{i=1}^{\infty} \theta^{-1} \circ \tau \circ \theta\left(\ell_{i}^{*} V^{*}\right)=\bigcup_{i=1}^{\infty} \tau^{*}\left(\ell_{i}^{*}\right) \tau^{*}\left(V^{*}\right),
$$

countably union of closed ets. Therefore $\tau^{*}\left(V^{*}\right)$ has non-void interior. Similarly, $\tau^{*-1}\left(V^{*}\right)$ also has non-void interior. We conclude $\tau^{*}$ is bicontinuous. And $\theta^{*}\left(\operatorname{Aut}\left(\mathscr{L}^{*}, \mathscr{D}^{*}\right)\right) \supset \mathrm{Aut}_{0} F$.

Proposition 12. Assume $[F, F]^{-}$is a uniform subgroup of $F$. Then Aut $_{0} F$ is an analytic group if and only if Aut $_{0} F$ is a Baire space, i.e. it satisfies the Baire Category Theorem. In particular, when $F$ is a second countable group, then Aut $_{0} F$ is an analytic group.

Proof. We keep all the notation from the discussion before this proposition. Let $\operatorname{Aut}_{1}\left(\mathscr{L}^{*}, \mathscr{D}^{*}\right)=\theta^{*-1}\left(\operatorname{Aut}_{0} F\right)$. Since $\operatorname{Aut}_{0}\left(\mathscr{L}^{*}, \mathscr{D}^{*}\right) \subset \operatorname{Aut}_{1}\left(\mathscr{L}^{*}, \mathscr{D}^{*}\right), \operatorname{Aut}_{1}\left(\mathscr{L}^{*}, \mathscr{D}^{*}\right)$ is a Lie group with countable many components. If Aut $_{0} F$ is a Baire space, then $\theta^{*}\left(\operatorname{Aut}_{0}\left(\mathscr{L}^{*}, \mathscr{D}^{*}\right)\right)=$ Aut $_{0} F$. Because $\theta^{*}$ is an injection, a fortiori, Aut ${ }_{0} F$ is an analytic group. Conversely since every locally compact topological group is a Baire space, so Aut $_{0} F$ is a Baire space when it is an analytic group. This finishes the proof of the first part of the proposition. Now, if $F$ is a second countable group, the compact-open topology on $\mathrm{Aut}_{0} F$ is complete. Since the Birkhoff topology on Aut ${ }_{0} F$ coincides with the compact-open topology [3], therefore $A_{u t} F$ is an analytic group in this case.

Remark 13. Let $G$ be a connected locally compact topological group. Let $L \times K$ be a local direct product of $G$. Let $\mathscr{L}=\bigcup_{n=1}^{\infty} L^{n}$. Let $\mathscr{L}^{*}$ be the analytic group obtained from

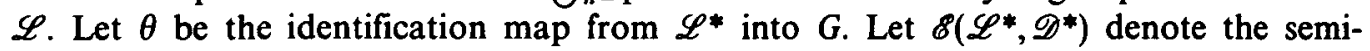
group of all the endomorphisms of $\mathscr{L}^{*}$ which leave $\mathscr{D}^{*}=\theta^{-1}(K \cap \mathscr{L})$ pointwise fixed. We can define an isomorphism $\theta^{*}$ from $\mathscr{E}\left(\mathscr{L}^{*}, \mathscr{D}^{*}\right)$ into $\mathscr{E}(G)$ by the rule $\tau^{*}=\tau$, where 
$\tau(\ell)=\theta\left(\tau^{*}\left(\ell^{*}\right)\right)$ for $\ell \in \mathscr{L}$ and $\tau(k)=k$. It was stated in [3] that $\theta^{*}$ is a bicontinuous isomorphism from $\mathscr{E}\left(\mathscr{L}^{*}, \mathscr{D}^{*}\right)$ onto its image. It is not difficult to see that $\theta^{*}$ is continuous. However, the proof of the continuity of $\theta^{*-1}$ in [3] seems incomplete. Especially, the argument given in line 14, p. 370 of [3] is not clear to us. Precisely, let $\bar{L}$ be a local Lie subgroup of $L$. Let $\tau(\bar{L}) \subset D \times L$. In general, it is unclear why $\tau(\bar{L})$ has to be inside $L$. One sufficient condition for this to be true is that $D$ is totally disconnected (cf. [7]). Even if we assume that $\tau(\mathscr{L}) \subset \mathscr{L}, \mathscr{L}=\bigcup_{n=1}^{\infty} L^{n}$, in general, $L \neq \mathscr{L} \cap D$, so one cannot always draw the conclusion $\tau(\bar{L}) \subset L$.

Proof of the main theorem. (I) If $\mathrm{Aut}_{0} G$ is locally compact, then $\mathrm{Aut}_{0} F$ is locally compact by Corollary 4 . If $A^{4} t_{0} F$ is locally compact, then either $\operatorname{dim} F$ is finite or $F /[F, F]^{-}$is compact by Lemma 6 . As we remarked before, Aut $_{0} F$ is a Baire space when it is locally compact. This finishes the proof in one direction.

(II) If $\operatorname{dim} Q<\infty$, then $\mathrm{Aut}_{0} F$ is an analytic group by Lemma 5 . Aut ${ }_{0} G$ is locally compact by Corollary 4 . Now in the case $\operatorname{dim} Q$ is not finite, then $G /[G, G]^{-}$is compact by assumption. Then $F /[F, F]^{-}$is compact. By Proposition $12, \mathrm{Aut}_{0} F$ is an analytic group. $A$ fortiori, Aut $_{0} G$ is locally compact.

Now, we shall study the problem: when Aut $G$ is locally compact. We keep all the notation from previous discussions.

Lemma 15. $G=F S$ where $S$ is the (almost-direct) product of a family of compact simple analytic subgroups of the maximal compact normal subgroup $K$ of $G$ and $F$ is the identity component of the centralizer of $K$.

Proof. By Lemma $1, G=F K$. Since $G / F$ is connected, so $G=F K_{0}$. Since $K_{0}$ is a compact-connected group, $K_{0}=A S$ where $A$ is the identity component of the centre of $K$ and $S$ the product of a family of compact simple analytic subgroups. Hence $G=F A S=F S$ since $A \subset F$.

Let $Q_{1}=Q \cap[F, F]^{-}$. Let $\phi$ be a continuous homomorphism from $G$ into $Q_{1}$. Define the automorphism $\tau_{\phi}$ on $G$ by $\tau_{\phi}(x)=x \phi(x)$ for $x \in G$. Let $\phi\left(G ; Q_{1}\right)=$ $\left\{\tau_{\phi}: \phi \in \operatorname{Hom}\left(G, Q_{1}\right)\right\}$.

Lemma 16. $\left.\phi G ; Q_{1}\right)$ is a closed normal subgroup of Aut $G$.

Proof. Given any $\phi \in \operatorname{Hom}\left(G ; Q_{1}\right), \phi$ is trivial on $[G, G]^{-}=[F, F]^{-} S$. Let $\sigma \in$ Aut $G$. Then $\sigma \tau_{\phi} \phi^{-1}(x)=\sigma\left(\sigma^{-1}(x) \phi\left(\sigma^{-1}(x)\right)=x \sigma \phi \sigma^{-1}(x) \in \phi\left(G ; Q_{1}\right)\right.$ since $Q_{1}$ is a characteristic subgroup of $G$ and $\sigma \phi \sigma^{-1} \in \operatorname{Hom}\left(G, Q_{1}\right) . \phi\left(G ; Q_{1}\right)$ is a normal subgroup of Aut $G$. Now let $\tau_{\phi_{n}}$ be a net converging to $\tau$. Then $\lim \tau_{\phi_{n}}(x)=\lim x \phi_{n}(x)=\tau(x), \lim \tau_{\phi_{n}}(x)=$ $\lim x \phi_{n}(x)=\tau(x)$, for $x \in G$. Then $x^{-1} \tau(x)=\lim \phi_{n}(x) \in Q_{1}$. Define $\phi(x)=x^{-1} \tau(x)$. Therefore $\phi=\lim \phi_{n}$ and $\phi \in \operatorname{Hom}\left(G ; Q_{1}\right)$. 
Let $\pi$ be the canonical homomorphism from $G$ onto $G / Q_{1} S$. Then $\pi$ induces an homomorphism $\pi^{*}$ : Aut $G \rightarrow$ Aut $G / Q_{1} S$.

Lemma 17. Let $\tau$ be an automorphism of $G$ which is in the kernel of $\pi^{*}$. Then $\tau=\tau_{1} \tau_{2}$ with $\tau_{1} \in \phi\left(G, Q_{1}\right)$ and $\tau_{2}$ leaves $F$ point-wise fixed, $\tau_{1} \tau_{2}=\tau_{2} \tau_{1}$.

Proof. Since $\tau\left(x Q_{1} S\right)=x Q_{1} S, x^{-1} \tau(x) \in Q_{1} S$ for all $x \in G$. Let $x \in F$. Then $x^{-1} \tau(x) \in F$ since $F$ is a characteristic subgroup of $G$. Therefore $x^{-1} \tau(x) \in F \cap Q_{1} S=Q_{1}(F \cap S)$. Since $F \cap S$ is totally disconnected and $\left\{x \tau^{-1}(x) \mid x \in F\right\}$ is connected, hence $x^{-1} \tau(x) \in$ the identity component of $Q_{1}$. In particular, this shows that $\phi(x)=x^{-1} \tau(x)$ is a homomorphism from $F$ into $Q_{1}$ and $\tau(x)=x \phi(x)$ for $x \in F$. Now let $x \in F \cap S$. Then $x^{-1} \tau(x) \in F \cap S$. As noted before, $F \cap S$ is totally disconnected. But $\left\{x^{-1} \tau(x): x \in F\right\} \subset$ identity component of $Q_{1}$. This implies that $x^{-1} \tau(x)=1$ when $x \in F \cap S$. Hence we can extend $\phi$ to $F S$ simply by defining $\phi(S)=1$. Let $\tau_{1}=\tau_{\phi}$. Then $\tau_{2}=\tau_{1}^{-1} \tau$ is an automorphism such that $\tau_{2}(x)=x$ for all $x \in F$. It is clear that $\tau=\tau_{1} \tau_{2}=\tau_{2} \tau_{1}$. Now the proof of the lemma is complete.

Proposition 18. Let $G$ be a connected locally compact group. Assume that $G /[G, G]^{-}$is a finite-dimensional group. Let $G=F S$ where $F$ is the identity component of the centralizer of the maximal compact normal subgroup $K$ of $G$ and $S$ is the semi-simple part of $K$. Let $Q$ be the compact part of the centre of $G$. Then Aut $G$ is locally compact if and only if the following conditions hold:

(1) $\operatorname{Hom}\left(G, Q_{1}\right)$ is locally compact where $Q_{1}=Q \cap[F, F]^{-}$.

(2) Aut $(S, Z(S))$ is locally compact, where $Z(S)$ is the centre of $S$.

(3) $\left(\phi\left(G ; Q_{1}\right)\right.$ Aut $\left.(G ; F)\right)$ Aut $_{0} G$ is an open subgroup and it is a Baire space. Also Aut $_{0} G$ is a Baire space.

Proof. First, note Aut $(S, Z(S))$ can be identified with Aut $(G ; F)$ since every automorphism $\sigma$ of $S$ which leaves its centre pointwise fixed can be identified with an automorphism of $G$ simple by defining $\sigma(x)=x$ when $x \in F$. Now we proceed to the proof.

(I) Necessity. Let $\pi$ be the canonical homomorphism from $G$ onto $G / Q_{1} S$. Let $\pi^{*}$ be the homomorphism from Aut $G$ into Aut $G / Q_{1} S$ induced by $\pi$. Since $Q_{1}$ is the maximal compact normal subgroup of $[F, F]^{-},[F, F]^{-} / Q_{1}$ is an analytic group. By assumption, $G /[G, G]^{-}=G /[F, F]^{-} S$ is a finite-dimensional group, so $G / Q_{1} S$ is a finite-dimensional group. Aut $G / Q_{1} S$ is a Lie group by Lemma 7 . Hence Aut ${ }_{0} G / Q_{1} S$ is an open analytic subgroup of Aut $G / Q_{1} S$. If Aut $G$ is locally compact, then $\pi^{*-1}\left(A_{u t} G / Q_{1} S\right)$ is an open subgroup of Aut $G$. This implies that $\left(\operatorname{Aut}_{0} G\right)\left(\operatorname{ker} \pi^{*}\right)$ is an open subgroup of Aut $G$. So we know $\left(\phi\left(G ; Q_{1}\right)\right.$ Aut $\left.(G ; F)\right)\left(\right.$ Aut $\left._{0} G\right)$ must be an open subgroup. It is locally compact, so it is a Baire space. Conditions (1) and (2) also follow immediately since they are closed subgroups of locally compact groups.

(II) Sufficiency. By condition (3), Aut ${ }_{0} G$ is a locally compact $\sigma$-compact group. 
Let $\phi \Delta=\phi\left(G ; Q_{1}\right)$ Aut $(G ; F)$. Then we have a continuous isomorphism from Aut $_{0} G /\left(\right.$ Aut $\left._{0} G\right) \cap \phi$ onto $\phi$ Aut $_{0} G / \phi$. Since $A \mathrm{At}_{0} G / \phi \cap \mathrm{Aut}_{0} G$ is $\sigma$-compact and locally compact and $\phi \mathrm{Aut}_{0} G / \phi$ is a Baire space, hence $\phi \mathrm{Aut}_{0} G / \phi$ is locally compact. Since $\phi \cong \phi\left(G ; Q_{1}\right) \times$ Aut $(G ; F)$, it is locally compact. Therefore $\phi$ Aut $_{0} G$ is locally compact. By assumption, it is an open subgroup of Aut $G$. Therefore Aut $G$ is locally compact.

We say $G$ has property $(C)$ if there is a local direct product $L \times D$ of $F$ such that $[\mathscr{L}, \mathscr{L}]$ is closed in $\mathscr{L}$ where $\mathscr{L}=\bigcup_{n=1}^{\infty} L^{n}$.

Proposition 19. Assume $G$ has property $(C)$. Let $M$ be a maximal compact subgroup of $G$. Let $B(M)$ be the subgroup of Aut $G$ consisting of all the automorphisms of $G$ which leave $M$ invariant. Then Aut $G=\left(\mathrm{Aut}_{0} G\right) B(M) . B(M)$ is locally compact if and only if there exists a compact subgroup $\phi$ of $B(M)$ such that $\phi B_{0}(M)$ is an open subgroup of $B(M)$ and $\phi$ leaves $[F, F]^{-}$pointwise fixed. Here $B_{0}(M)$ is the identity component of $B(M)$.

Proof. Given any $\tau \in$ Aut $G$, there exists an inner automorphism $I_{x}$ such that $I_{x}(M)=\tau(M)$. A fortiori, $I_{x}^{-1} \circ \tau \in B(M)$. Since $I_{x} \in$ Aut $_{0} G$, therefore Aut $G=\left(\right.$ Aut $\left._{0} G\right) B(M)$. Assume now that $B(M)$ is locally compact. Then there exists a compact, totally disconnected subgroup $\phi^{\prime}$ such that $\phi^{\prime} B_{0}(G)$ is an open subgroup of $B(M)$. (This is a fact from the general structure theorems of locally compact groups.) Choose a local direct product $L \times D$ such that (1) $[\mathscr{L}, \mathscr{L}]$ is closed in $\mathscr{L}$ where $\mathscr{L}=\bigcup_{n=1}^{\infty} L^{n}$; (2) $L \times D$ is $\phi^{\prime}$ invariant. Then $[\mathscr{L}, \mathscr{L}]$ is a characteristic subgroup so it is Aut $G$ invariant. The group $[\mathscr{L}, \mathscr{L}]$ can be given an analytic group topology which will be denoted by $[\mathscr{L}, \mathscr{L}]^{*}$. Let $r$ be the restriction map: Aut $G \rightarrow$ Aut $[F, F]^{-}, r(\tau)=\tau \mid[F, F]^{-}$. Since $r(\tau)$ leaves $[\mathscr{L}, \mathscr{L}]$ invariant, $r(\tau)$ defines an automorphism $r(\tau)^{*}$ of $[\mathscr{L}, \mathscr{L}]^{*}$. Let Aut $_{1}[\mathscr{L}, \mathscr{L}]^{*}$ denote the subgroup $\left\{r(\tau)^{*}: \tau \in\right.$ Aut $G$ and $\tau$ leaves $D$ invariant $\}$. We show that Aut $_{1}[\mathscr{L}, \mathscr{L}]^{*}$ is a closed subgroup of the Lie group Aut $[\mathscr{L}, \mathscr{L}]^{*}$. Let $\Gamma^{*} \theta^{-1}(\Gamma)$ where $\Gamma=D \cap[\mathscr{L}, \mathscr{L}]$ and $\theta$ is the identification map from $[\mathscr{L}, \mathscr{L}]^{*}$ onto $[\mathscr{L}, \mathscr{L}]$. Then $\Gamma^{*}$ is a discrete central subgroup. Let $\operatorname{Aut}\left([\mathscr{L}, \mathscr{L}]^{*}, \Gamma^{*}\right)$ be the group of automorphisms of $[\mathscr{L}, \mathscr{L}]^{*}$ which leaves $\Gamma^{*}$ pointwise fixed. Let Aut $\sim\left([\mathscr{L}, \mathscr{L}]^{*}, \Gamma^{*}\right)$ be the subgroup of Aut $[\mathscr{L}, \mathscr{L}]^{*}$ which leaves $\Gamma^{*}$ invariant. Then Aut $\left([\mathscr{L}, \mathscr{L}]^{*}, \Gamma^{*}\right) \subset \mathrm{Aut}_{1}[\mathscr{L}, \mathscr{L}]^{*} \subset$ Aut $^{\sim}$ $\left([\mathscr{L}, \mathscr{L}]^{*}, \Gamma^{*}\right)$. Because Aut $\left([\mathscr{L}, \mathscr{L}]^{*}, \Gamma^{*}\right)$ is an open subgroup of Aut $^{\sim}\left([\mathscr{L}, \mathscr{L}]^{*}, \Gamma^{*}\right)$, Aut $_{1}[\mathscr{L}, \mathscr{L}]^{*}$ is a Lie group. Let $\theta^{*}$ be the injection from $\operatorname{Aut}_{1}[\mathscr{L}, \mathscr{L}]^{*}$ into Aut $[F, F]^{*}$. Since $r\left(\phi^{\prime}\right)$ is a compact, totally disconnected subgroup in $\theta^{*}\left(\right.$ Aut $\left._{1}[\mathscr{L}, \mathscr{L}]^{*}\right)$, a fortiori, it is a Lie group. Therefore we have a subgroup $\phi$ of finite-index in $\phi^{\prime}$ such that $r(\phi)$ is the trivial subgroup. It is clear $B_{0}(M) \phi$ is open in $B(M)$. Now the proof of the proposition is complete.

Remark 20. When Aut $G$ is locally compact, then $\mathrm{Aut}_{0} G$ and $B(M)$ are both locally compact. Conversely, when $A_{u t} G$ and $B(M)$ are locally compact, in general we do not know if Aut $G$ is locally compact or not. It is clear that, when $G$ is a second countable locally compact group, then Aut $G$ is Baire space. Aut $G$ is locally compact when $B(M)$ is locally compact by a standard Baire categorical argument. 
Observe that the subgroups $\phi\left(G ; Q_{1}\right)$ and $\operatorname{Aut}(G, F)$ defined in Proposition 19 are subgroups of $B(M)$. In the general case, i.e., without the assumption that $G /[G, G]^{-}$is finite-dimensional, $\operatorname{dim} Q / Q_{1}$ may be infinite. $Q$ could be very complicated. Our present knowledge on the automorphisms of compact, connected abelian groups is not enough to give a further satisfactory characterization of $B(M)$.

\section{REFERENCES}

1. P. Chen and T. S. Wu, On the automorphism groups of locally compact groups and on a theorem of M. Goto, Tamkang J. Math. 17 (1986), 99-116.

2. P. Chen and T. S. Wu, On a class of topological groups, Math. Ann. 266 (1984), 499-506.

3. M. Goto and N. Kimura, Semigroup of endomorphisms of a locally compact group, Trans. Amer. Math. Soc. 87 (1958), 359-371.

4. G. Hochschild, The Structure of Lie Groups (Holden-Day, Inc., San Francisco, 1965).

5. S. Helgason, Differential Geometry Lie Groups and Symmetric Spaces (Academic Press, New York, 1978).

6. K. Iwasawa, On some types of topological groups, Ann. of Math. 50 (1949), 507-558.

7. D. H. LEE and T. S. $W_{U}$, On the group of automorphisms of a finite-dimensional topological group, Michigan Math. J. 15 (1968), 321-324.

8. D. Mongomery and L. ZIPPIN, Topological Transformation Groups (Interscience Tracts in Pure and Applied Math., Second Printing, New York, 1964).

9. A. Gleason and R. Palais, On a class of transformation groups, Amer. J. Math. 799 (1957), 631-648.

10. M. Moskowirz, Homological algebra in locally compact abelian groups, Trans. Amer. Math. Soc. 127 (1967), 361-404.

11. J. Peter and T. Sund, Automorphisms of locally compact groups, Pacific J. Math. 76 (1978), 143-156.

12. T. S. Wu, Closure of Lie subgroups and almost periodic groups, Bull. Inst. Math. Acad. Sinica 14 (1986), 325-347.

Case Western Reserve University

Cleveland, Ohio 44106

USA 